we find the explanation of the fact established by statistics, that the operation of paracentesis thoracis has been attended with more success in cases of empyema than in cases of acute pleurisy with serous effusion. We conceive a close analogy to exist between the results of an operation performed on an individual in vigorous health, who, perhaps, may have met with an accident peremptorily requiring immediate operation, and on one who, after having been long the subject of wasting disease, has to undergo a no less serious one, and the results of the operation of paracentesis thoracis in acute pleurisy with serous effusion, and in empyema. And, as the surgeon is more sanguine as to the result of his operation performed on the subject whose health has been run down by previous wasting illness, than on him who, up to the time of his accident requiring operation, has been in full health-so statistics have proved that the physician has more reason to anticipate a successful result to his operation in empyema than in acute pleurisy. The explanation of this would appear to be that, as the higher the animal is in the scale of beings the less tolerant is he of injury; an inferior animal will bear an amount of injury that would be fatal to man. Disease has the effect of degrading the higher animal, and bringing him down to a lower condition. But with this degraded condition he also acquires the superior patience of injury; or perhaps it might be said of him, that the conditions of his existence are now more simple; so that disease thus may be said to make some compensation for its other effects. Acute pleurisy surprises one commonly in the midst of strong health. Operation in this case resembles operation on one who, in full vigour, has been the subject of accident requiring immediate operation, while the condition of the system in empyema generally resembles that which has been brought down by long disease, so it resembles it in having come into a condition better suited to bear an injury; for we may regard an operation in the light of an injury, although undertaken for a salutary end and object.

ArT. X.-Operations for Strangulated Hernia, and their Results. By Jostay Smyly, A.B., F.R.C.S.I., Surgeon to the Meath Hospital and County of Dublin Infirmary.

TeE following cases are drawn up from notes which $I$ have preserved; and, although all the cases $I$ have operated upon are not 
given, yet, as no selection has been made, they may be fairly considered to embrace my experience. Certainly no case has been designedly omitted because its termination was unfavourable.

\section{FORM OF HERNIA.}

Of the 15 cases given, eight were those of inguinal, and occurred in males; and seven of femoral hernia, all in females. Nine of the cases recovered perfectly-viz., three of inguinal and six of femoral hernia. Of the six cases which terminated fatally five were inguinal hernia.

DURATION OF SYMPTOMS BEFORE OPERATION IN FATAL CASES.

With regard to the time the intestine has been subjected to strangulation, I find that No. 4 was operated upon four hours after the symptoms set in, and proved fatal 36 hours after the operation. No. 5 was operated upon nine hours after strangulation, and survived for 26 hours. No. 6 was operated upon 24 hours after strangulation, and survived 26 hours. No. 7 was operated upon 53 hours after strangulation, and survived six days. In No. 3, the strangulation existed for six days, and the patient survived about three weeks. No. 15, the sixth fatal case, was one of entero-epiplocele, a femoral hernia; strangulated for 36 hours, and the patient died the fifth day after operation. All the fatal cases were hospital patients.

CAUSE OF DEATH IN FATAL CASES.

In all the fatal cases peritonitis was the cause of death. In No. 7 the peritonitis was kept up by persistent strangulation and sloughing of the strangulated intestine; and in No. 4 the peritonitis was caused by extravasation of the contents of the intestine, the part which had been constricted having ulcerated through, and perforation having thus taken place. In case No. 15 the peritonitis was evidently caused by the omentum having been left in the sac. In case No. 2 the thickened omentum was cut away; and in case No. 8 it was returned into the cavity of the abdomen; and both these cases did well.

The success attending the cutting away of the thickened omentum bears out the truth of the statement of Sir A. Cooper, Mr. Laurence, and Mr. Teale, that excision of the omentum, and ligature of its vessels, is a mode of treatment " unattended with danger." 


\section{SEAT OF STRICTURE.}

In five of the cases of femoral hernia the stricture was relieved by cutting Gimbernaut's ligament; in one the stricture was caused by the cribriform fascia.

No. 14 shows the importance of observing the rule to avoid any sawing motion in dividing the stricture, and simply to press the knife against the stricture till it gives way. In No. 8 the seat of stricture was in the body of the tumour, and the constriction was caused by the contraction of what was formerly the neck of a sac. The case shows how a new sac may form above an old one, the old sac being pushed further down into the scrotum. It shows how the neck of a sac can become a constricting agent independently of muscular contraction, or the pressure of tendinous rings or fasciæ.

\section{AGE OF PATIENTS.}

The ages of those who recovered after operation were-males, $20,50,62$; females, $20,24,70,80,58,35$.

The ages of those who died were-males, 70, 32, 40, 45, 59; female, 34 .

\section{CHARACTER OF THE SAC.}

The strength of the sac is found to vary considerably. No. 3 is an example of a hernial sac of great thickness.

Case I.-Strangulated Inguinal Hernia; Operated on upon the Third Day of its Strangulation; Recovery.-P.L., aged 20, a servant, subject to reducible inguinal hernia on right side. On Friday he was heaving a large stone, when he felt severe pain in his rupture. $\mathrm{He}$ was unable to reduce it. Various means were used by the village doctor, but unsuccessfully; amongst others, croton oil was given to force a passage. On Sunday I found him in intense agony; he had his buttocks up against the wall, and was resting on his neck and shoulders. No impression could be made on the tumour by the taxis. The usual operation was immediately performed, and the sac opened into. A large quantity of fluid was found in it, and a small knuckle of intestine. When this was returned, a considerable quantity of serum flowed out from the cavity of the peritoneum.

Some hours after the operation the patient was bled, and was put upon calomel and opium, by which means the inflammatory symptoms were relieved, and he made a good recovery. 
Case II.-Strangulated Entero-Epiplocele; Inguinal Hernia; Operation; Recovery.-P. D., aged 62 years, a labourer, has had a reducible inguinal hernia, on the right side, for the last 10 years; he has worn a badly-fitting truss. He was admitted into the Meath Hospital, September 14th, '62, with the symptoms of strangulation, which had existed for the last 24 hours. A turpentine injection was immediately administered, 30 drops of solution of morphia given, and the taxis tried, assisted by a warm bath, at 103 degrees of temperature, and patient put under the influence of chloroform. All having failed, the operation was performed at half-past four o'clock, p.m. On opening the sac, a considerable quantity of darkcoloured fluid was discharged. The chief bulk of the tumour was found to be composed of thickened omentum, which surrounded the strangulated intestine. On freeing the stricture, the intestine was easily returned, but the omentum was so thickened and enlarged as to prevent its reduction. Having secured the neck of the omental tumour with a dressing forceps, the mass was cut away; the arteries were tied with fine silk, the ligatures being left long. The truncated portion was allowed to remain in the neck of the sac; the lips of the wound were brought together by three points of suture, \&c.; calomel and opium ordered. Seventeen hours after the operation, it was reported that he had a good night; no vomiting.

Sept. 22nd. Abscess has formed in the scrotum, which was opened this day. 28th. Two more abscesses have formed.

Oct. 6th. Able to wear a truss, and to sit up.

Nov. 12 th. Have just heard that $D$. is quite restored to health.

\section{CASE III.-Strangulated Inguinal Hernia; a Very Thickened} Sac a source of Error; Death.-J. H., aged 70, for some time subject to reducible inguinal hernia on right side. He never wore a truss. The intestine became incarcerated on Monday, Jan. 22nd, 1848 ; on the 25 th he was sent from the country, and admitted into the Meath Hospital. The taxis, assisted by O'Beirne's long tube, large cupping glasses applied to abdomen and pulled so as to make a tendency to a vacuum, turpentine enemata, \&c., made a very considerable alteration in the shape and size of the tumour. My impression was that the intestine was reduced, but that omentum remained strangulated. A warm bath was ordered, and afterwards ice to the tumour. 26th. Patient vomited last night, but not since; bowels have not been freed; he is delirious; the tongue is coated white, and brownish in the centre; pulse frequent. In 
consultation, at 10 o'clock, it was determined to postpone operating till $3 \mathrm{o}$ 'clock. It was the opinion of all that the symptoms were not now depending upon strangulated intestine, and that the tumour contained omentum only. At 4 o'clock it was agreed upon to operate. The sac was found to be enormously thickened; it contained nothing but serum; the lower part of the sac was cut away; its walls were half an inch thick; the wound was closed by sutures.

Feb. 3rd. Eighth day after operation. Tongue cleaning; wound suppurating; a small spot of the skin of the scrotum is sloughing. $12 \mathrm{th}$. Wound healing. 13th. Left the hospital, being anxious to return home.

I have since learned that $H$. gradually sunk, and died some days after getting home.

CASE IV.-Strangulated Inguinal Hernia; Operation Four Hours after Strangulation; Death, by Peritonitis, Thirty-four Hours after the Operation.-M. C., aged 32, has had reducible inguinal hernia for the last 15 months. At 8 o'clock, p.m., got a fall, which caused a larger protrusion of intestine than usual to take place; this was attended with great pain. He was carried to the Meath Hospital an hour after the occurrence; when a warm bath was given, blood was taken from the arm, and cupping glasses applied to the abdomen. A tobacco enema was given to assist the taxis. I saw him at midnight, and found him in a state of collapse from the tobacco enema, but suffering intense pain from the hernia. Entreating that he should be "cut open," or anything else done that would give him relief, the operation was then performed. Much difficulty was experienced in returning the intestine; it was distended with gas. The contents of the sac consisted entirely of small intestine. Two arteries being tied, the wound was closed by sutures, adhesive plaster compress, and bandage; 20 drops of Battley's sedative were given, but immediately after rejected from the stomach. One grain of opium in a pill ordered. The following day the report was most favourable. He got immediate relief after the operation. There was no tension nor tenderness of the abdomen; no vomiting nor hiccup; bowels had acted freely ; tongue rather white; great thirst; pulse 100 . In the afternoon, 15 hours after the operation, great pain and tenderness of the abdomen set in, with vomiting. Venesection was performed; calomel and opium ordered; a drachm of mercurial ointment placed in the axillæ, and a blister applied to the abdomen. Third day, it was reported that he was much relieved 
by the bleeding; but this morning he was found in a collapsed and dying state; he expired at 10 o'clock, a.m., 34 hours after the operation. On post mortem examination, it was found that the intestine, where it had been strangulated, had sloughed, and a small perforation had taken place, which was the immediate cause of the peritonitis. The sac was found distended by fluid, which led to the supposition, during life, that the intestine had again descended.

Case V.-Inguinal Hernia on Right Side; Strangulated only for Nine Hours when Operation was Performed; Died Twenty-six Hours afterwards of Peritonitis.-A man, aged about 40, subject to reducible inguinal hernia, on right side, for the last 14 years. Having gone to bed in a state of intoxication last night, he was roused, at three o'clock this morning, with symptoms of strangulated hernia. $\mathrm{He}$ was brought to the Meath Hospital at six o'clock, a.m., January 9th, 1845. At half-past 12 o'clock, noon, nine hours after first symptoms appeared, the taxis, and all means to assist it, having failed, the operation was performed, and the protruded intestine (which was intensely red) was reduced. There being evidence of general peritonitis, the patient was bled from the arm, in the afternoon, and calomel and opium given. Notwithstanding all the efforts that were made, the patient sunk, and died in the evening. On post mortem examination extensive peritonitis was discovered; no cause, except the previous strangulation, could be found.

CASE VI._Strangulated Inguinal Hernia; Operation performed Twenty-four Hours after Strangulation; Fatal in Thirty Hours.A man, aged 45 years, subject to reducible inguinal hernia for two years, which became irreducible on December 24th, 1845 . He was treated by a practitioner, for an enlarged gland, for 24 hours. On the 25th he was brought to the Meath Hospital. The taxis having failed, the operation was performed at 12 o'clock, noon. 26th. Considerable abdominal tenderness; inclination to vomit; pulse frequent; anxious countenance; ordered 12 leeches to the abdomen, a blister to the epigastrium, and a turpentine enema to be given immediately.

December $27 \mathrm{th}$, he died, 30 hours after the operation, of peritonitis.

Case VII.-Strangulated Inguinal Hernia; the Seat of Strangulation not Discovered; Sloughing of the Intestine; Peritonitis; 
Death.-W. I., aged 59, a coachman. At noon, on the 5th Jan., 1846 , while riding one horse and leading another, the one on which he was riding stumbled, and the other darted forward, and dragged him forcibly against the pommel of the saddle. He made a violent effort to pull up the falling horse, and felt a "giving way, accompanied with a tearing pain, and spasms in the lower part of the abdomen." On the 7th he was brought to the Meath Hospital. The taxis, assisted by venesection to 30 ounces, tobacco enema, \&c., having failed, it was resolved upon to operate at five o'clock, p.m. The sac was found thickened. It, to the surprise of all, contained nothing but dark, tarry, and fetid blood. The ring was divided; the patient expressed himself as having obtained relief; the finger was introduced into the cavity of the abdomen, and turned all round, but no intestine could be discovered connected with the ring. The wound was closed; calomel and opium ordered to be given every three hours. The symptoms of strangulation continued unabated-viz., the hiccup and stercoraceous vomiting. On the night of the 13th he died. On post mortern examination there was evidence of intense peritonitis ; effusion of lymph coating the intestines; of a bloody fluid in the cavity of the peritoneum; the intestines were found distended with flatus; a small knuckle of intestine, completely sphacelated, was found in a pouch behind the cord, in the situation of hernia by direct descent; it was quite concealed by the old hernial sac. At the operation it eluded the observation of the most experienced and discerning of my colleagues.

CASE VIII.-Strangulated Inguinal Hernia; Stricture in the Body of the Sac; Operation Forty-eight Hours after Strangulation; Recovery.-R. M., aged 50, a hairdresser, has had a reducible inguinal hernia, on the right side, for nine months. It came on in consequence of over-exertion while running.

On March 8th, 1841, symptoms of strangulation set in. Patient had eaten bacon and cabbage for dinner the day previous. When admitted into the Meath Hospital he was exceedingly low and faint; pulse was small and frequent; countenance pale and sunken; had frequent vomiting and hiccup; O'Beirne's long tube and other means to assist the taxis were employed; but, as no impression could be made upon the tumour, the operation was performed at 11 o'clock, a.m., on the 10th. This case presented a peculiarity, inasmuch as the stricture was found in the body of the sac, between its superior and middle third; the intestine above the constriction 
was soft and reducible, that below was thickened, and felt solid; it was highly vascular, and its surface coated with lymph; there was a deep depression between the sound and strangulated intestine; there was a considerable portion of fatty and thickened omentum, as well as intestine, in the sac; it required some manœuvring to reduce the protrusions. The wound was closed with four points of suture; a compress and bandage were applied. In four hours reaction was established; 10 ounces of blood were taken from the arm, and a draught given, with 20 drops of acetum opii, immediately after the operation; two grains of calomel and half a grain of opium to be given every two hours.

11th. Going on well; quite free from pain, vomiting, \&c.; countenance calm; pulse 80 .

This patient recovered, but was liable to protrusions of the intestine. Two years after his recovery he had an attack of strangulation. He was seen seven hours after the first symptom appeared; and the hernia was reduced by the taxis, assisted by the application of ice to the tumour, and a turpentine enema. The most decided advantage was derived from acetate of morphia, half a grain given every half hour.

The state of the case was very unpromising at first. The tumour was so large that it could not be grasped in the two hands; it was extremely hard, and very painful, and patient had frequent vomiting. However, after the second dose of morphia, the whole was replaced.

Case IX.-Strangulated Femoral Hernia, Right Side; Operation; Recovery.-Miss C., aged 20, has had a hernia for some months. She was told by a medical man that the tumour in her groin was an enlarged gland. She paid no particular attention to it. One evening she was seized with pain in the tumour, followed by vomiting, and immediately sent for assistance. The taxis, assisted by the application of ice, \&c., having been tried during the night, next morning, in consultation, and assisted by Mr. Cusack, the operation was performed, 12 hours after symptoms of strangulation set in. The patient recovered without a bad symptom.

\section{Case X.-Strangulated Femoral Hernia; Stricture Divided} without Opening the Sac; Cure.-M. R., aged 24, servant, subject to crural hernia, on right side, for last two years. She states, that during this period it had become incarcerated six times; and that having suffered violent vomitings for eight or 
10 hours, the protrusion returned spontaneously. She was admitted into the Meath Hospital on the morning of the 14th of January, 1837, in a state of collapse; pulse 60, feeble; vomiting occasionally; intense pain both in the tumour and in the abdomen. Ice was applied to the tumour, and large exhausted cupping glasses to the abdomen, by means of which traction was used so as to elevate the parietes, and draw in, as it were, the hernial tumour. The taxis having failed, and hiccup having come on, it was determined, in consultation, to operate. On the cribriform fascia being divided, above and below the tumour, it was manifest that the constriction was relieved; gentle pressure was employed, and the intestine was returned, without opening into the sac. The patient expressed herself as freed from all uneasiness the instant the intestine was replaced.

The wound was dressed in the ordinary way, and the patient made a rapid recovery.

CaSE XI.-Strangulated Femoral Hernia; Operation; Re. covery.-Mrs. F., aged 70 years, subject to reducible femoral hernia, right side, for the last 20 years. She has worn a truss. On the 5 th of February, 1858, a small knuckle of intestine became incarcerated; on the 8 th severe vomiting set in. All attempts at reduction having proved useless, on the 10th February the operation was performed. The stercoraceous vomiting continued for some time after she was removed to her bed; the symptoms gradually subsided; the bowels acted well on the 11 th. She made a good recovery.

\section{Case XII.-Femoral Hernia; Strangulated for about Thirty} Hours; Operation; Cure.-Miss W., aged 80, affected with bronchitis; of regular habits, and very temperate; subject, for some time, to reducible femoral hernia, but never wore a truss. On the 22nd of March, 1851, the protruded intestine became strangulated. Next morning bilious vomitings set in, when she sent for assistance. Terebinthinate enemata were administered, and ice was applied to the tumour. The taxis was persevered with for seven hours. The ordinary operation, by opening the sac, was then performed; when visited, four hours after, she was free from vomiting and pain; the pulse was still frequent; small doses of calomel and opium were ordered. On the 13th day after she was sitting up-the wound nearly quite healed. Miss W. lived for many years, and had no return of the hernia.

vor. $\mathrm{x} \times x \mathrm{x}$, No. 70, N. s. 
Case XIII.-Strangulated Femoral Hernia; Operation; Cure.Mrs. W., aged 58, subject to reducible femoral hernia on left side, for some years. She wore an inefficient truss. The hernia had been strangulated for 22 hours. The taxis, assisted by tobacco enemata, having failed, the operation was performed. There was an immense quantity of fat external to the sac, which rendered the operation difficult. The stricture, at the neck of the sack, being divided, the bowel, which was of a dark livid colour, was returned. Immediate relief of all the symptoms followed the operation. The patient made a good recovery.

Case XIV.-Strangulated Femoral Hernia, unattended by Constipation; Vermiform Appendix in the Sac; Operation; Recovery.Mrs. G., the wife of a physician, aged 35, having eaten greens at dinner, was seized with colicky pains in the stomach at night. In the morning she perceived, for the first time, a tumour in the right groin; she had no vomiting, but nausea; she, that evening, took aperient pills, which operated in the night. May 31st, 36 hours after first symptom set in, sent for assistance. The tumour, which was of an oblong shape, and not as large as an egg, was quite irreducible. Ice was applied. June 1st., calomel, followed by sulph. of magnesia, given; bowels were affected. The taxis having failed, the operation was performed at seven o'clock, p.m. The sac was found full of bloody serum. The vermiform appendix alone was found strangulated. This case did well; there was neither fever nor inflammation after the operation. She made a rapid recovery.

Case XV.-Entero-Epiplocele; Femoral Hernia; Operation; Omentum Left in Sac; Peritonitis; Death on Fifth Day.-A female, aged 34 , subject to reducible hernia for 16 years. The operation was performed on the third day after the strangulation took place. Besides a knuckle of intestine, a considerable portion of omentum, converted into a solid mass, was found adhering to the sac, and caused much difficulty in discovering its neck. The stricture being freed, and the intestine returned, it was determined upon to leave the omentum in the sac. The wound was closed by sutures, \&c.; calomel and opium were ordered, and leeches to the abdomen. Third day after operation-no tenderness on pressure over the abdomen, nor any symptom of peritonitis. Fourth day-peritonitis has set in ; ordered 20 leeches, a blister to the abdomen, and calomel and opium. She died on the fifth day after the operation.

Post mortem examination.-A large quantity of purulent matter 
was found in the cavity of the peritoneum; the portion of intestine which had been strangulated had so recovered as not to be distinguished from the other intestines; the omentum in the sac was much inflamed, and was evidently the source from which the peritonitis sprung.

It was remarkable in this case that the obturator artery was given off from the epigastric, and ran a great risk of being wounded. It must have been pushed upwards on the bistoury used as a wedge. Had a sawing motion been given to the instrument the artery must have been cut; it was found crossing the wound in the fascia, just as the cross stroke in the letter $A$.

ArT. XI.-On the Causes and Treatment of Closure and Immobility of the Jaws. By Christopher Heath, F.R.C.S., Assistant Surgeon to, and Lecturer on Anatomy at, the Westminster Hospital.

Cases of permanent closure of the jaws from cicatrices within the mouth, \&c., are not of very rare occurrence; but their description and treatment seem to have been very generally neglected by modern English authors. Erichsen, Pirrie, Druitt, and Skey make no mention of the affection; and Samuel Cooper, in the last edition of his Surgical Dictionary which he revised, merely refers to a case treated by Valentine Mott, who, in 1831, operated on a case of sloughing of the cheek, with subsequent closure of the jaws, by transplanting a piece of skin; but he enters no further into the treatment. In the new edition of Cooper's Dictionary, 1861, Vol. I, the only passage $I$ can find, bearing on the question, is the following, under the head of "Cicatrization":-

"In the mouth, after sloughing of the cheek and gums from profuse salivation, the cicatrized surface is so rigid as scarcely to allow of the separation of the teeth, but it becomes more pliant in time." This latter statement, however, is not borne out by general experience.

Mr. Fergusson, in the last edition of his Practical Surgery, p. 602, says:-

"The lower jaw occasionally becomes so closely bound to the upper, that the teeth cannot be sufficiently separated to admit of solid food. This condition may arise from inflammation and 